\title{
Models of Quasi-Periodic Variability in Neutron Stars and Black Holes
}

\author{
D. Psaltis ${ }^{1}$ \\ ${ }^{1}$ Center for Space Research, Massachusetts Institute of Technology, Cambridge, MA 02139, USA
}

\begin{abstract}
Black holes and weakly-magnetic accreting neutron stars show strong quasi-periodic variability over timescales that span a very wide range. This property offer us a unique opportunity to improve our understanding of basic physical processes in strong gravitational fields as it reveals, for the first time, phenomena that occur on the most fundamental timescales near accreting compact objects. In this article, I review our current understanding of the variability properties of accretion flows onto neutron stars and black holes. I discuss a number of challenges faced by theoretical models, in order to identify open questions in accretion physics that need to be addressed. Finally, I discuss the relation to and implications for variability models of the detection of nearly coherent oscillations during type I X-ray bursts in several neutron-star sources.
\end{abstract}

\section{INTRODUCTION}

It is generally accepted that matter is accreted onto compact objects at the high observed rates because of the turbulent transport of angular momentum. Since turbulent flows are typically variable, it is not surprising that the X-ray brightness of even the so-called steadily accreting objects is observed to show significant $(\sim 10-30 \%)$ fluctuations over a large range of timescales, from milliseconds to weeks.

The range of variability frequencies in an accretion flow is determined by the extent of the flow itself, as well as by the specific mechanism that produces them. As an example of the latter, in a geometrically thin accretion disk, the motion of the gas is primarily in the azimuthal direction, the flow of angular momentum is in the radial direction, whereas the propagation of photons is in the vertical direction. Depending on what causes the variability, the accretion luminosity might, therefore, be modulated at any or all of the three dynamical timescales that correspond to each of these directions. All characteristic frequencies in an accretion flow depend strongly on the distance from the central object. As a result, for a process occurring throughout an accretion disk, one would expect strong variability at all timescales, from milliseconds to days, corresponding to the innermost and outermost parts of the accretion disk, respectively. Indeed, the power spectra of accreting compact objects show broad-band variability components at all these timescales. However, the most prominent and striking feature of their power spectra is the presence of a small number of characteristic frequencies, that appear in the form of narrow, quasi-periodic oscillation (QPO) peaks.

Because of this observational fact, the aim of current models is the identification of characteristic radii in the accretion flow that pick only a small range of frequencies and hence produce narrow QPO peaks in the power spectra. Such radii include the radius of the innermost stable circular orbit (which is important in the diskoseismic models of, e.g., Wagoner 2000), the magnetospheric radius (Alpar \& Shaham 1985), or the sonic-point radius (Miller, Lamb, \& Psaltis 1998). The simultaneous presence of multiple QPOs is then attributed to the interaction of the flow with the stellar spin (as in, e.g., Miller et al. 1998), to the presence of multiple characteristic radii in the accretion flow (as discussed, e.g., in Miller et al. 1998), or to different characteristic frequencies occurring at the same region in the flow, as in the case of the diskoseismic models (see Wagoner 2000) and the relativistic models of Stella et al. (1999) and Psaltis \& Norman (2000).

Addressing which of all the characteristic frequencies or radii is responsible for the observed variability properties of accreting neutron stars and black holes is a subject of active research. In particular, three important issues and their implications for theoretical models are still a matter of debate: the importance 
of the modulation of the accretion flow at the stellar spin frequency (as required by beat-frequency models, in the case of accretion onto neutron stars; see Miller et al. 1998), the suggested similarity between the variability properties of neutron-stars and black holes (Psaltis et al. 1999), and the relation of variability models with the spin-frequency interpretation of nearly coherent oscillations observed during type I X-ray bursts (Strohmayer 2000, these proceedings).

\section{MODELS OF THE PERSISTENT VARIABILITY OF ACCRETING COMPACT OBJECTS}

The motion of a fluid element in an accretion flow is determined by the combined effect of several forces that act on it, such as gravity, magnetic forces, radiation forces, and viscosity. The properties of the different interactions allow us to define different timescales (or frequencies), at which the various forces operate, and radii, at which the effects of these interactions are dominant. A number of characteristic frequencies and radii that are often invoked in models of the variability properties of compact objects are discussed below.

\section{Characteristic Variability Frequencies}

A simple estimate of the three characteristic dynamical frequencies at distance $r$ away from a compact object is given by the three corresponding test-particle frequencies: the orbital frequency in the azimuthal direction, the epicyclic frequency in the radial direction, and the vertical oscillation frequency. These correspond to the characteristic frequencies of oscillation of the gas density in a ring of non-interacting particles orbiting around a compact object, when perturbed in the azimuthal, radial, and vertical directions, respectively. For a rotating compact object with an external Kerr spacetime, they have three distinct and non-zero values, given by (see, e.g., Perez et al. 1997)

$\Omega^{2}=\frac{M}{r^{3}\left[1 \pm \alpha_{*}\left(M / r^{3}\right)^{1 / 2}\right]}$,

$\kappa^{2}=\Omega^{2}\left[1-6 M / r \pm 8 \alpha_{*}\left(M / r^{3}\right)^{1 / 2}-3 \alpha_{*}^{2} / r^{2}\right]$,

and

$\Omega_{\perp}^{2}=\Omega^{2}\left[1 \mp 4 \alpha_{*}\left(M / r^{3}\right)^{1 / 2}+3 \alpha_{*}^{2} / r^{2}\right]$,

respectively. Here, $M$ is the mass and $\alpha_{*}$ the specific angular momentum per unit mass of the compact object, and the fundamental constants are set to $c=G=1$. All three frequencies describe periodic phenomena, which can reach a very high coherence when confined to a narrow range of radii. Moreover, the azimuthal orbital frequency (11) is the highest of all characteristic frequencies and is, therefore, expected to describe the fastest phenomena occurring in the accretion flows. For these reasons, all current models have invoked one or all of the above as the fundamental frequencies of variability of accreting compact objects.

An accretion flow, however, is a hydrodynamic system and hence the test-particle frequencies (11)-(3) can only be approximations to the frequencies at which the flow is modulated. In the case of a geometrically thin accretion disk, additional relevant timescales are (see, e.g., Frank et al. 1992), the sound-crossing time given by

$t_{\mathrm{c}} \equiv \frac{c_{\mathrm{s}}}{r} \simeq\left(\frac{r}{h}\right) \frac{1}{f_{\mathrm{K}}}$,

where $c_{\mathrm{S}}$ is the sound speed and $h$ is the scale height of the disk, and the viscous timescale, which is given by

$t_{\mathrm{V}}=\frac{1}{\alpha_{\mathrm{SS}}}\left(\frac{r}{h}\right)^{2} \frac{1}{f_{\mathrm{K}}}$,

where $\alpha_{S S}$ is the viscosity parameter, and measures the time it takes for a density inhomogeneity to be smoothed by viscosity. The last two timescales do not describe periodic phenomena and, therefore, are not expected to directly determine any characteristic frequency of quasi-periodic variability. However, the effects they describe, such as the propagation of sound waves or the viscous dissipation of density perturbations, can alter the frequencies of oscillations in the accretion flows as well as determine their lifetimes, and hence their coherence (see, e.g., Wagoner 2000; Psaltis 2000). Including such hydrodynamic effects is both necessary for models to be physically self-consistent and required by all current models for achieving agreement with observations (see, e.g., Lamb \& Miller 2000; Psaltis 2000). 


\section{Characteristic Radii}

The observed QPOs in both neutron-star and black-hole systems are typically narrow, with fractional widths as low as $\delta \nu / \nu \sim 10^{-2}$ (van der Klis 2000). Because all characteristic frequencies in an accretion flow have a strong dependence on radius and height above the equatorial plane, such small fractional widths severely localize the physical mechanism that determines the QPO frequencies to a narrow annulus in a geometrically thin component of the accretion flow, so that $\delta r / r \leq \delta \nu / \nu \sim 10^{-2}$ and $h / r \leq(\delta \nu / \nu)^{1 / 2} \sim 10^{-1}$.

The notion of a narrow annulus in an accretion disk at a characteristic radius, across which the fluid properties change considerably, is not new in accretion theory and several such radii have been identified so far. For example, in the case of an accretion disk around a black hole or a high-mass neutron star, the Keplerian flow is terminated at the radius of the innermost stable circular orbit, inside which the flow becomes quasi-radial and supersonic (see, however, Hawley \& Krolik 2000). This radius depends only on the mass and spin of the central object and, therefore, the dynamical frequencies that correspond to it are very stable. This stability has been used both in constructing models for QPOs in black-hole systems with frequencies that show very little dependence on accretion rate (see, e.g., Wagoner 2000) and in placing model-independent upper bounds on the frequencies of other variable-frequency QPOs in both black-hole and neutron-star systems (see, e.g., Miller et al. 1998).

The presence of variable-frequency QPOs, however, in most neutron-star and black-hole systems requires the presence of a characteristic annulus with a radius that depends on accretion rate or any other variable parameter of the system. For example, in the case of a neutron star with a dynamically important magnetic field, the Keplerian flow can be terminated at the so-called magnetospheric radius at which magnetic torques can remove the angular momentum of the accreting gas in a narrow boundary layer and bring it to corrotation with the star. The radius of the boundary layer depends on the mass accretion rate through the inner disk (see, e.g., Ghosh \& Lamb 1978) and can become comparable to the neutron-star radius for weak magnetic fields and high accretion rates. This radius has been used in the magnetospheric beat-frequency models of the $\sim 20-60 \mathrm{~Hz}$ QPOs observed from several neutron stars (see, e.g., Alpar \& Shaham 1985).

The absence of any direct evidence of magnetic truncation of the accretion disks around neutron stars that show high-frequency variability as well as the existence of variable-frequency QPOs around black holes necessitates the presence of characteristic radii that can be generated and sustained without requiring a central object with a hard surface or an anchored magnetic field. Radiation drag forces from an external illuminating source can also remove efficiently the angular momentum of the accreting gas, producing a sharp transition from Keplerian to quasi-radial inflow (see, e.g., Miller et al. 1998). This, so-called sonic point radius, can exist only very close to the compact object $(\sim 3-10$ Schwarzschild radii $)$ and is invoked for setting the variability frequencies in the sonic-point beat-frequency model of the high-frequency QPOs observed from neutron stars. As another example, the transition in the thermal properties of an unstable accretion disk during a dwarf-nova like instability cycle is very sharp and has been used in models of nearlycoherent variable-frequency oscillations observed from white dwarf systems (see discussion below).

In summary, the requirements for a geometrically thin accretion disk with a sharp transition in its properties are rather model independent. They arise from three properties of the QPOs observed in both neutron-star and black-hole systems: the fact that their frequencies are highly variable, while, at the same time, they are relatively coherent and strictly reproducible with spectral state. These requirements challenge our current understanding of accretion flows around compact objects. The nature of variable characteristic radii is not well understood and requires hydrodynamic models of the accretion disks that take into account external forces besides gravity. Moreover, the requirement for a geometrically thin accretion disk close to a compact object accreting at near-Eddington accretion rates (as in the case of the luminous source Sco X-1, which shows the most coherent oscillations at the highest inferred accretion rates) requires the construction of accretion disk models at high accretion rates that are both geometrically thin and stable, in contrast to our current understanding (see, however, Abramowicz 1985).

\section{kHz QPOs: A signature of Spin-Orbit Interaction or of Relativistic Effects?}

The most thoroughly studied of the variability phenomena observed from an accreting compact object are the $\mathrm{kHz}$ QPOs discovered with the Rossi X-ray Timing Explorer in the lightcurves of many neutron-star systems. These are twin QPO peaks with variable frequencies in the $\simeq 200-1200 \mathrm{~Hz}$ range and a peak 

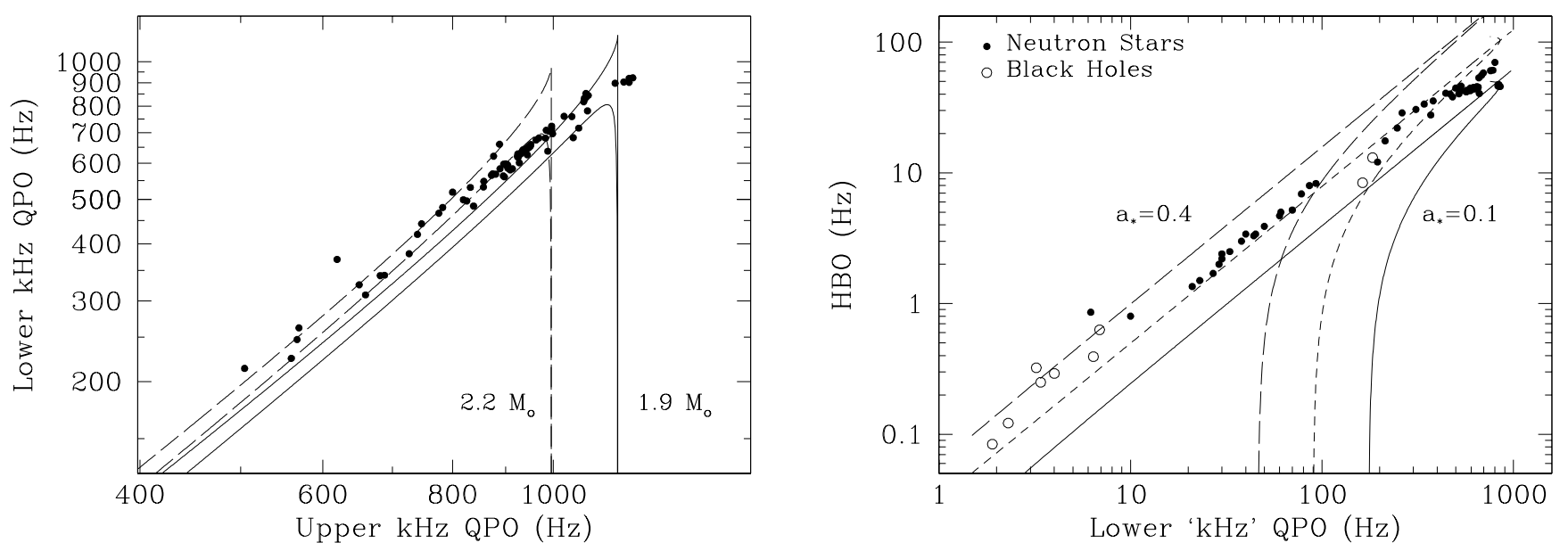

Fig. 1. Correlations of QPO frequencies in neutron-star and black-hole systems. The curves are the predictions of the model in which all the QPO frequencies are attributed to general relativistic frequencies in the accretion flow (for details see Psaltis \& Norman 2000).

separation of $\simeq 250-350 \mathrm{~Hz}$ (see van der Klis 2000 for a review).

In the early observations, the peak separation of the two $\mathrm{kHz}$ QPOs appeared to be constant and equal to the frequency of highly-coherent oscillations observed during thermonuclear bursts from the same sources (see below and Strohmayer 2000, these proceedings). This property gave rise to the beat-frequency models of $\mathrm{kHz}$ QPOs (Strohmayer et al. 1996; Miller et al. 1998) according to which the upper kHz QPO occurs at the Keplerian frequency at a characteristic radius in the accretion disk (the magnetospheric or the sonic-point radius) and the lower $\mathrm{kHz}$ QPO occurs at the beat between the frequency of the upper $\mathrm{kHz} \mathrm{QPO}$ and the neutron-star spin frequency. Beat-frequency models rely on the interaction between the orbital motion of the accreting gas and the stellar spin through magnetic or radiation forces and can account, by construction, for the similarity of the peak separation of $\mathrm{kHz}$ QPOs with the frequencies of burst oscillations. However, they are challenged by the subsequent discovery that the peak separation of the $\mathrm{kHz}$ QPOs in all sources strongly decreases with increasing QPO frequency (but see Lamb \& Miller 2000) and, moreover, by the possibility that the same type of variability phenomena occur also in black-hole systems, for which a beat-frequency model cannot be viable (see Psaltis et al. 1999).

Detailed studies of three simultaneously detected QPOs in both neutron-star and black-hole systems revealed that their frequencies follow a small number of tight correlations that span up to three orders of magnitude in frequency (Psaltis et al. 1999 and Fig. 1; in the case of neutron stars, the three QPOs are the upper $\mathrm{kHz}$ QPO, the lower $\mathrm{kHz} \mathrm{QPO}$, and a low-frequency, $\sim 20-60 \mathrm{~Hz}$, QPO previously known). This property led to the construction of the so-called relativistic models, in which the three QPO frequencies correspond to the Keplerian, the periastron precession, and the nodal precession frequencies of perturbed orbits in a characteristic radius in the accretion disks (Stella, Vietri, \& Morsink 1999; Psaltis \& Norman 2000). Relativistic models can account for the presence of similar variability phenomena in both neutronstar and black-hole systems and their observed frequency correlations (see Fig. 1). However, they do not address directly the similarity of the peak separation of $\mathrm{kHz}$ QPOs with the frequencies of burst oscillations.

An observable signature of each theoretical model is the spectrum of characteristic frequencies it predicts beyond the ones currently detected (see, e.g., Miller 2000b). For this reason, the inadequacy of current data to distinguish between different models can be overcome with the detection of additional QPO peaks. Even though some attempts for detecting expected peaks at different frequencies have given negative results (Méndez \& van der Klis 2000a), a third kHz QPO has been recently discovered in three low-luminosity sources (Jonker, Méndez, \& van der Klis 2000). Its properties were shown to favor the relativistic model of $\mathrm{kHz}$ QPOs (Psaltis 2000), even though its consistence with a beat-frequency model cannot be ruled out (see, e.g., Alpar 1986; Jonker et al. 2000)

The fundamental difference, however, between the two categories of theoretical models of neutron-star 

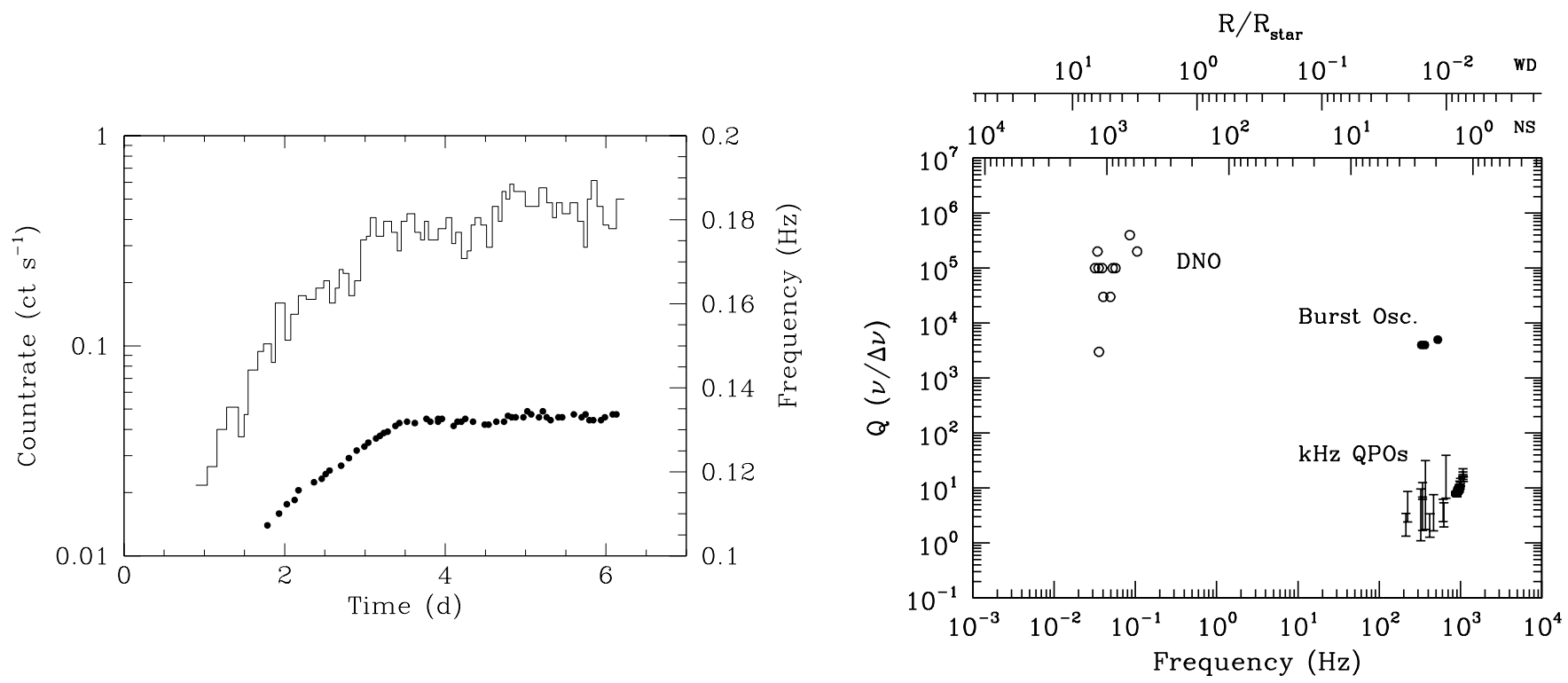

Fig. 2. (Left) Evolution of the EUV countrate and the frequency of the dwarf-nova oscillations (DNO) in SS Cyg (Mauche 1996). (Right) Coherence of various observed oscillations; the $\mathrm{kHz}$ QPOs correspond to the $Z$ sources, the burst oscillations to KS 1731-260, 4 U 1702-90, and $4 \mathrm{U} 1728-34$, and the DNO have been taken from the compilation of Patterson (1981). The top axis shows the radius at which the orbital frequency is equal to the observed frequency, for a $0.5 M_{\odot}$ white dwarf $\left(R_{\text {star }}=10^{8} \mathrm{~cm}\right)$ and a $2 M_{\odot}$ neutron $\operatorname{star}\left(R_{\text {star }}=10^{6} \mathrm{~cm}\right)$.

variability discussed above is the role of the neutron-star spin frequency in determining some of the observed QPO frequencies. None of the known $\mathrm{kHz}$ QPO sources has shown so far pulsations at the neutron-star spin frequency in the persistent emission. The only evidence for the magnitude of the spin frequencies of sources that show persistent $\mathrm{kHz}$ QPOs comes from the modeling of the highly coherent oscillations observed during thermonuclear bursts. In the following section, I discuss the interpretation of burst oscillations and its implications for models of the persistent variability of neutron stars.

\section{BURST OSCILLATIONS: INTERPRETATION AND IMPLICATIONS}

A large number of accreting neutron stars show recurrent type I X-ray bursts that are characterized by fast $(\sim 1 \mathrm{~s})$ rise and slower exponential decay (Lewin et al. 1996) and are thought to be the result of thermonuclear flashes on the surfaces of the neutron stars. Their lightcurves are expected to be modulated at the stellar spin frequencies because of the anticipated anisotropy of the thermonuclear burning. Fast timing observations of accreting neutron stars during thermonuclear bursts indeed showed highly coherent signals at $\sim 300-600 \mathrm{~Hz}$ that have been interpreted as occurring at frequencies that are within a percent of the stellar spin frequencies (see review by Strohmayer 2000, these proceedings).

\section{Spin-Frequency Interpretation}

The spin-frequency interpretation of burst oscillations is motivated mainly by two of their observed properties: their stability and coherence. First, burst oscillations from the same neutron star source have been shown to occur always at frequencies within a percent of each other, even between bursts that were months apart. Second, when the slow frequency evolution of burst oscillations that occurs at timescales of seconds is removed, the coherence of the resulting oscillations is large compared to any other QPO observed in the same systems, by about an order of magnitude. Given that the spin frequency of a neutron star is highly stable and coherent, it is, therefore, natural to assume that burst oscillations occur at the stellar spin frequencies.

It is important to note, however, that the above arguments are neither strong nor conclusive. For example, there exist several other frequencies in the systems that are as stable as the stellar spin frequency, such as the Keplerian frequency at the innermost stable circular orbit, or the maximum radial epicyclic frequency. The coherence $\left(Q \equiv \nu / \delta \nu \sim 10^{3}\right)$ of the burst oscillations might also be higher than any other known QPO 


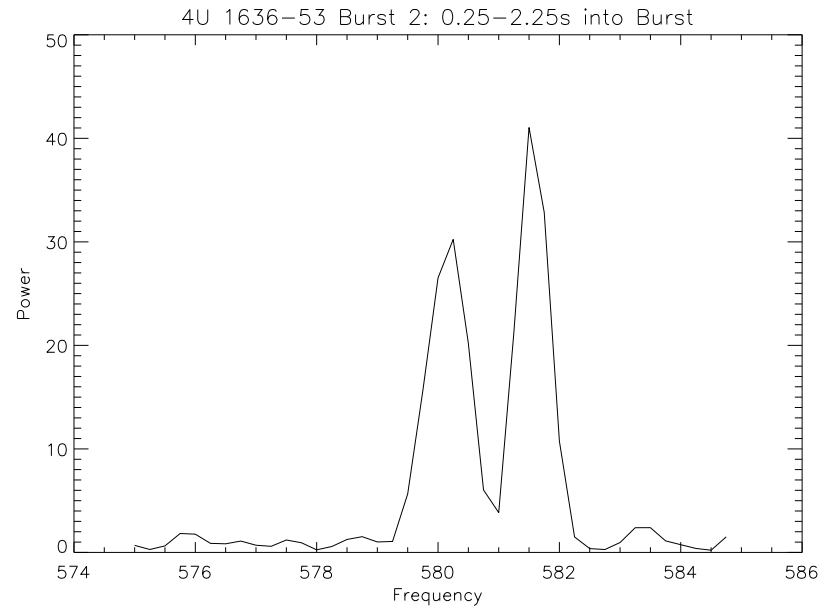

Fig. 3. (Left) Detail of the power spectrum of the first two seconds of a burst observed from the source $4 \mathrm{U} 1636-53$, showing the simultaneous presence of two distinct oscillation peaks; these peaks are also seen simultaneously in the dynamical power spectra and therefore cannot be due to the rapid evolution of one oscillation frequency (see, e.g., Miller 2000a). (Right) Amplitudes of the lower (filled symbols) and upper (open symbols) kHz QPOs in (a) 4U 1728-34 and (b) $4 U 1608-52$ as a function of their frequencies (from Méndez et al. 2000b).

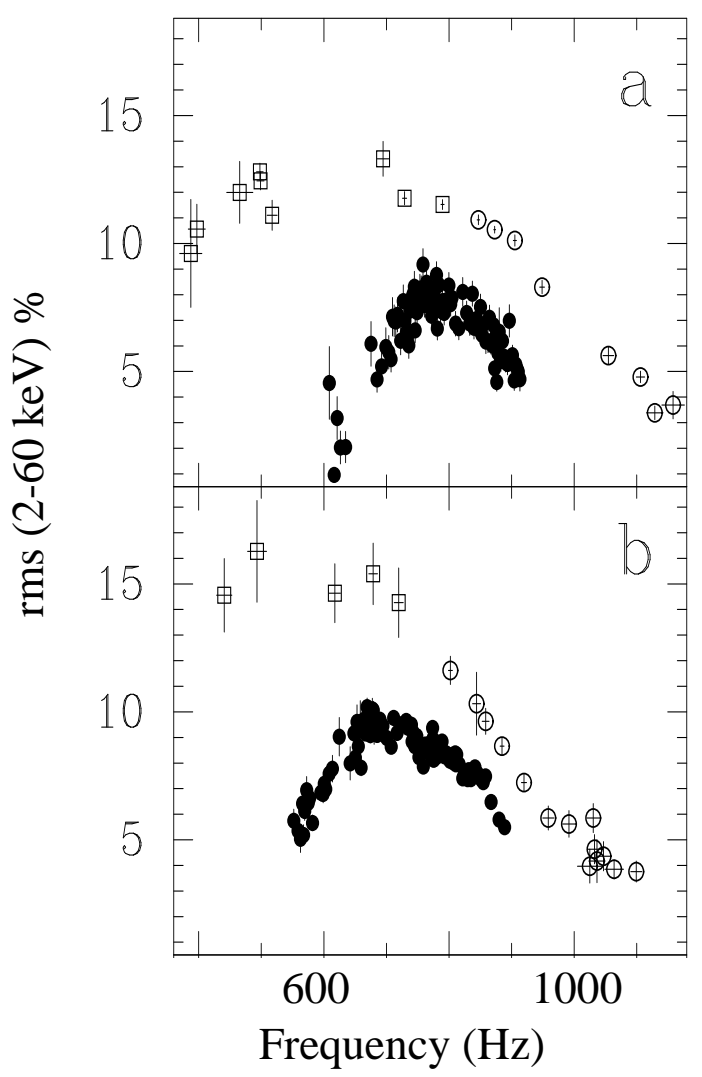

in neutron-star systems, but is not comparable to the coherence of the periodic pulsations of rotation- or accretion-powered pulsars (even though this might be just due to the limits on the coherence imposed by the short lifetime of the oscillations). Moreover, QPOs with significantly higher coherences $\left(Q \simeq 10^{4}-10^{6}\right)$ have been detected in the high-energy emission of several accreting white-dwarf systems (the so-called dwarf-nova oscillations; see, e.g., Patterson 1981). The properties of dwarf-nova oscillations are strikingly similar to those of burst oscillations (see Fig. 2). Their frequencies are variable at timescales of days and scale with inferred luminosity, so that they cannot be related to the white-dwarf spin-frequencies but are probably phenomena in the accretion flows. When their slow frequency evolution is removed, the coherence of the oscillations can reach values as high as $10^{6}$. Finally, their frequencies are comparable to the dynamical timescale at a few white-dwarf radii, as is the case of burst-oscillation frequencies in neutron stars (see Fig. 2). None of these arguments suggests that dwarf-nova oscillations and burst oscillations are produced by the same mechanism. They show, however, that accretion disk phenomena can produce periodic oscillations of the accretion flows very close to the central objects, with very high coherences.

The spin-frequency interpretation of burst oscillations faces also a number of additional challenges. For example, the stringent upper limits on the amplitudes of any harmonics of the fundamental frequencies strongly contradict model predictions. More importantly, the detection of burst oscillations with amplitudes as high as $10 \%$ at the tails of the bursts requires that the burning front has ignited only $\sim 30 \%$ of the neutron star surface, even several (10-20) seconds after the start of the burst, which is hard to understand. Finally, the interpretation of the frequency evolution of burst oscillations in terms of a decoupling of the burning layer from the rest of the star cannot account for the large (up to 1.3\%; Galloway et al. 2000) observed fractional change of the oscillation frequencies. The interpretation of the frequency evolution of burst oscillations becomes also more complicated, given the fact that in a number of bursts, multiple oscillation peaks have been detected simultaneously with comparable yet distinct frequencies (see, e.g., Miller 2000a; Galloway et al. 2000; see also Fig. 3).

Despite all these issues, the detection of oscillations during type I X-ray bursts from the millisecond pulsar SAX J1808.4-3086 at the known spin-frequency (in't Zand et al. 2000) provides strong support to the spin-frequency interpretation of burst oscillations. It is possible that the oscillations observed during type I bursts from this source are the anticipated signatures of non-uniform burning on the neutron-star 
surface (given that the millisecond pulsar is the only known, weakly-magnetic accreting neutron star with a non-uniform surface emission during the persistent emission), whereas the burst oscillations observed from other sources that show no pulsations in their persistent emission is not. Conclusive identification of the oscillations observed in SAX J1808.4-3086 as bona fide burst oscillations or detection of the neutron stars spin frequencies in the persistent emission of sources that show burst oscillations will settle the issues discussed above.

\section{Implications for Beat-Frequency Models}

The spin-frequency interpretation of burst oscillations provides the motivation for and the strongest argument in favor of the beat-frequency interpretation of the $\mathrm{kHz}$ QPOs in neutron-star systems. However, in some sources burst oscillations are detected with fractional amplitudes that reach $\sim 100 \%$ while the upper limits of any coherent pulsations in the persistent emission of the same sources are 2-3 orders of magnitude smaller. It is still puzzling how the asymmetry during thermonuclear burning can produce such strong oscillations at the stellar spin frequency (so that the compactness and orientation of the system are not unfavorable), the asymmetry during the persistent emission can produce a strong beat-frequency QPO between a Keplerian frequency and the stellar spin (so that the accretion flow interacts with the stellar spin), and yet the persistent emission is not modulated at the stellar spin frequency.

In several sources, the maximum peak separation of the $\mathrm{kHz}$ QPOs is comparable (to within several percent) to the frequency of burst oscillations or to half of that frequency. This observational fact together with the spin-frequency interpretation of the burst oscillations require that either (a) the peak separation of the $\mathrm{kHz}$ QPOs is always comparable to the stellar spin frequency and the burst oscillations occur at the spin frequency or its overtone or (b) the burst oscillations occur always at the stellar spin frequency and the peak separation of the $\mathrm{kHz}$ QPOs is comparable to the spin frequency or its sub-harmonic. Note that the above requirement needs to be addressed by any model of the $\mathrm{kHz}$ QPOs; the beat-frequency interpretation requires that option (a) is correct.

Identifying, however, the frequency of the $\sim 600 \mathrm{~Hz}$ burst oscillations with the first overtone of the stellar spin frequency, as required by beat-frequency models, is hard to justify. Such an identification would require, in some sources, the simultaneous ignition of thermonuclear burning on two, almost exactly antipodal spots on the stellar surface, presumably its magnetic poles. The frequency evolution and coherence of such burst oscillations would necessitate that the burning layers at the two antipodal spots decouple from the rest of the star and are subject to the same differential rotation. Finally, the absence of any oscillations with comparable amplitudes at the postulated spin frequencies (i.e., at half the observed frequencies) would require fine tuning in the geometry of the systems (both ignition spots to lie on the rotational equator and the observer to view the systems very nearly edge-on) which has a very small a priori probability to occur and is, therefore, inconsistent with the fact that the majority of burst oscillation sources (six out of nine; see Muno et al. 2000) show burst oscillations at twice the peak separation of the $\mathrm{kHz}$ QPOs.

\section{Implications for Relativistic Models}

In the relativistic models of $\mathrm{kHz}$ QPOs, the spin frequency of the neutron star does not determine directly the frequencies of any of the QPOs. As a result, the spin-frequency interpretation of the burst oscillations does not have direct implications for such models. However, it is uncomfortable, within a relativistic model, to rely on pure coincidence in order to explain the observed similarity between the burst oscillation frequencies and the peak separations of the $\mathrm{kHz}$ QPOs or its harmonic. Moreover, the stellar spin frequency determines the properties of the spacetime and hence the magnitudes and correlations of the predicted QPO frequencies. As a result, measurements of the neutron-star spin frequencies constrain relativistic models in two ways.

First, the peak separation of the $\mathrm{kHz}$ QPOs, in the relativistic models, is comparable to the radial epicyclic frequency at a characteristic radius in the accretion disk. The spin-frequency interpretation of the burst oscillations would therefore require either (a) the spin frequencies of the neutron stars to evolve towards the maximum radial epicyclic frequency in the accretion flow, which is hard to justify, or (b) one or both of the $\mathrm{kHz}$ QPOs to attain their high observed amplitudes when their peak separation is comparable to the stellar spin frequency. The latter option is also motivated by the fact that the amplitude of the lower $\mathrm{kHz}$ QPO in several sources is observed to increase with frequency, reach a maximum, and decrease again 

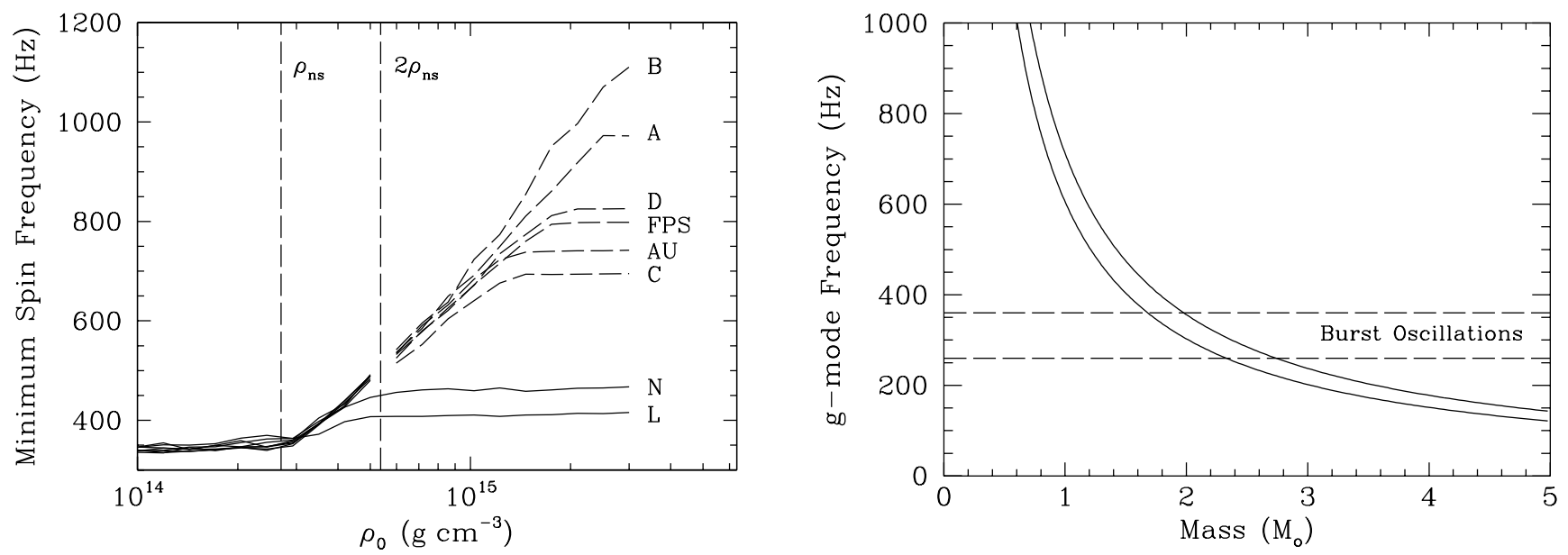

Fig. 4. (Left) Minimum neutron-star spin-frequency required by the relativistic model of QPOs, for different equations-of-state, if the observed low-frequency QPOs occur at the first overtone of the Lense-Thirring frequency. (Right) Range of predicted $g$-modes frequencies for a Kerr spacetime and different hydrodynamic corrections (for details see Wagoner 1999).

over a relatively narrow range of frequencies so that the QPO is undetectable when the predicted peak separation is significantly different than the burst oscillation frequency (see Fig. 3). Such a possibility can be accommodated in the hydrodynamic relativistic model of Psaltis \& Norman (2000), if the accretion flow at the characteristic radius is also perturbed at the stellar spin frequency in the radial direction, giving rise to a strong resonance only when the radial epicyclic frequency is comparable to the stellar spin..

Second, the frequency of the third $(\simeq 20-60 \mathrm{~Hz})$ QPO observed in neutron-star systems and identified with the nodal precession frequency in the relativistic models, is proportional to the spin frequencies of the neutron stars and their moments of inertia. It can be shown that for any equation of state of neutron-star matter, the observed QPO frequencies are too high to be produced around a neutron star that is spinning at the frequencies inferred from burst oscillations (Psaltis et al. 1999). This is also shown in Fig. 4, were different equations-of-state and densities (up to which a given equation-of-state is assumed to be valid) are used in inferring the minimum spin frequency required for the observed QPO frequencies to be equal to twice the nodal-precession frequencies in sources such as Sco X-1 and 4U 1728-34. In the latter source, the required $>500 \mathrm{~Hz}$ spin frequency is substantially higher than the $\sim 360 \mathrm{~Hz}$ spin frequency inferred from burst oscillations.

\section{An Alternative}

The only theoretical idea that has been developed in any detail so far for explaining the properties of the burst oscillations is that of a burning surface layer that can decouple from the rest of the star and rotate at a frequency comparable but not strictly equal to the neutron-star spin frequency. It will be instructive, however, if other possibilities are also explored and tested against observations. In fact, the relativistic models of the persistent-emission QPOs can be used as motivation to exploring models in which the burst oscillations are generated in the accretion flows and not on the stellar surfaces.

In the relativistic models, the peak separation of the $\mathrm{kHz}$ QPOs is comparable to the epicyclic frequency in a characteristic radius in the accretion flow. This frequency has a maximum at a radius very close to that of the innermost stable circular orbit that depends only on the mass and the spin of the compact object and is, therefore, very stable. Moreover, near this maximum, a frequency cavity is produced in which disk modes (the so-called $g$-modes) can be efficiently trapped (see Wagoner 2000). The $g$-modes can be excited when the thermonuclear flash disturbs the inner accretion flow and can last for a time comparable to the viscous timescale, which is of order of several seconds. The frequencies of $g$ - modes are comparable to the maximum epicyclic frequency in the accretion flow, are equal to the observed $\sim 300 \mathrm{~Hz}$ frequencies for neutron stars with masses $\sim 2 M_{\odot}$, and alleviate the need for most known weakly-magnetic neutron stars to be spinning at very similar frequencies. The frequencies of the lower-order modes are comparable to the maximum peak 

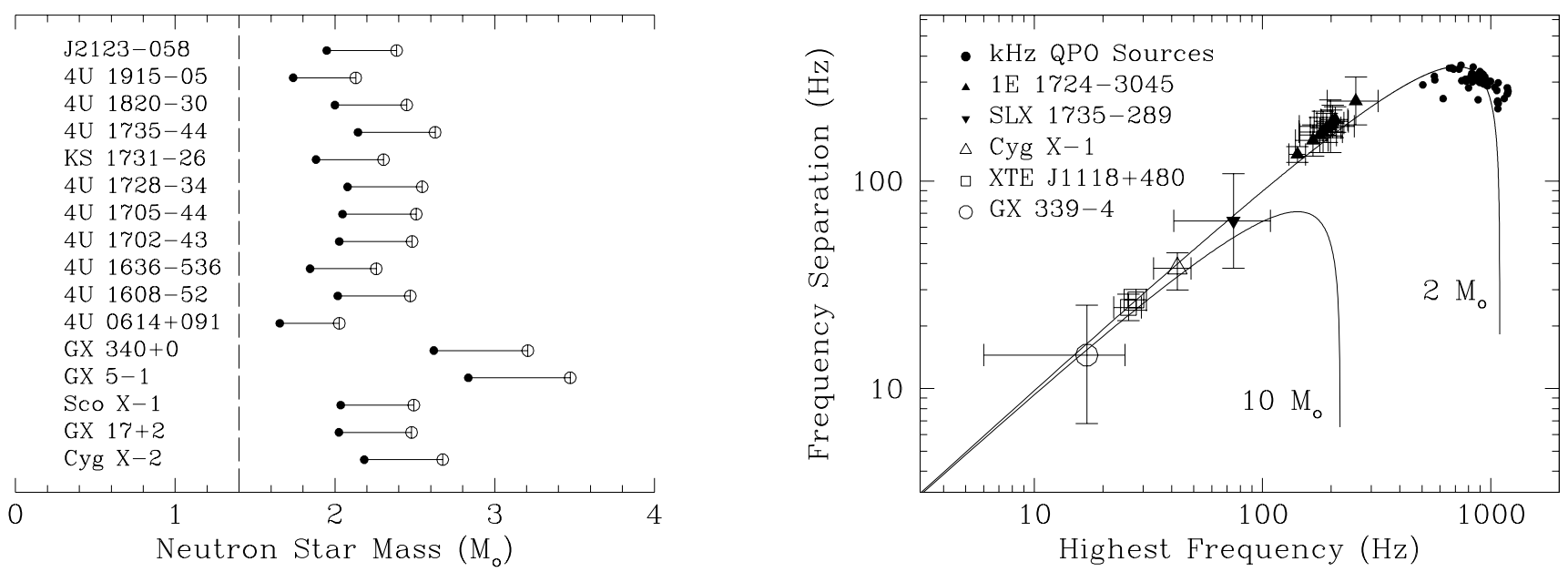

Fig. 5. (Left) Maximum neutron-star masses for individual sources allowed by the current observations; filled and open circles correspond to non- and slowly-rotating $\left(\alpha_{*}=0.3\right)$ stars (after Chakrabarty et al. 2000). (Right) Frequency separation of the highest observed frequencies in different neutron-star (filled symbols) and black-hole (open symbols) sources as a function of the highest observed frequency; the lines correspond to the predictions of the relativistic model (after Belloni et al. 2000).

separation of the $\mathrm{kHz}$ QPOs, as predicted by the relativistic models, whereas the frequency of the next higher-order mode is $\sim 4$ times larger and not harmonically related, which will account for the absence of harmonic structure in the observed power spectra of bursts. Moreover, theoretical calculations show that the oscillatory spectra of $g$-modes contain several closely spaced modes (Wagoner 2000), which would appear in the observed power spectra as the simultaneously detected peaks shown in Fig. 3. Finally, assuming that the accretion (and not only the burst) luminosity is modulated at the burst oscillation frequency, then the inferred fractional amplitudes of burst oscillations will be significantly smaller than currently reported.

It important, however, to stress that a disk-mode interpretation of the burst oscillations faces a number of challenges, as well. For example, even though accretion modes seem to be able to reach very high coherences (as observed, e.g., in the dwarf-nova oscillations), current studied of $g$-modes limit their coherence to values smaller than observed. More importantly, though, the conclusive identification of the oscillations observed in SAX J1808.4-3086 as bona fide burst oscillations will offer conclusive evidence against such interpretations.

\section{TESTING FUNDAMENTAL PHYSICS WITH COMPACT-OBJECT VARIABILITY}

Theoretical models of the variability properties of accreting neutron stars and black holes are still in their infancy. Important questions, such as the importance of the stellar spin or the steady-state structure of geometrically thin accretion disks at high accretion rates need to be addressed before the properties of timedependent accretion can be modeled in detail. However, even in their current state, models of the variability of accreting neutron stars and black holes show the potential of such studies in probing the equation of state of neutron-star matter beyond the reach of laboratory experiments and in searching general relativistic effects that occur only in the strong-field regime.

For a given neutron-star spin frequency, the equation of state determines the gravitational mass and radius of the neutron star and hence the Keplerian frequency at the innermost stable circular orbit. The latter is the highest possible frequency of any long-lived, quasi-coherent phenomenon produced in the accretion flow around the neutron star. It can, therefore, be used together with the highest observed QPO frequency in placing upper bounds on the mass of individual sources (Miller et al. 1998; see Fig. 5). This is a rather model-independent argument and is applicable to almost all current model of neutron-star variability. More specific comparison of particular models to the data lead to more severe constraints on the properties of neutron-star matter. For example, the observed correlations between QPO frequencies can be accounted for in the relativistic model only if the neutron-star masses are $>1.8 M_{\odot}$ and hence only if the equation of state is relatively stiff.

Finally, the high frequencies of the observed QPOs strongly suggest that they originate very close to the 
neutron-star surfaces or the event horizons of black holes. As a result, they must carry signatures of the strong gravitational fields in which they are produced, such as the presence of an innermost stable circular orbit outside the compact object or of a maximum in the radial epicyclic frequency. Evidence for both phenomena have already been reported in the literature (Zhang et al. 1998; Belloni et al. 2000; see also Fig. 5) and conclusive detection of them will provide us with the first direct evidence of strong-field general relativistic effects.

\section{ACKNOWLEDGEMENTS}

It is a pleasure to thank a number of people who contributed in many different ways to the work presented in this article. I would especially like to thank T. Belloni, M. van der Klis and the high-energy group at the Univ. of Amsterdam, as well as D. Chakrabarty, D. Galloway, M. Muno, and the MIT group on burst oscillations. I am also grateful to F. Özel for useful discussions and carefully reading the manuscript and to M. Muno for help in preparing several of the figures in this paper.

\section{REFERENCES}

Abramowicz, M. A., PASJ, 37, 727, 1985.

Alpar, M. A., MNRAS, 223, 469, 1986.

Alpar, M. A., and J. Shaham, Nature, 316, 239, 1985.

Belloni, T, D. Psaltis, and M. van der Klis, ApJ, submitted, 2000.

Chakrabarty, D., S. E. Thorsett, and D. Psaltis, in preparation, 2000.

Frank, J., A. R. King, and D. Raine, Accretion Power in Astrophysics Cambridge University Press, 1992.

Galloway, D., D. Chakrabarty, M. Muno, and P. Savov, ApJ, submitted (astro-ph/0010072), 2000.

Ghosh, P., and F. K. Lamb, ApJ, 232, 259, 1978.

Hawley, J., and J. Krolik, ApJ, in press (astro-ph/0006456), 2000.

in't Zand, J., et al., $A \mathscr{G} A$, submitted, 2000.

Jonker, P., M. Méndez, and M. van der Klis, ApJ, 540, L29, 2000.

Lamb, F. K., and M. C. Miller, ApJ, submitted (astro-ph/0007460), 2000.

Lewin, W. H. G., J. van Paradijs, and R. Taam, in X-ray Binaries, eds. W. H. G. Lewin, J. van Paradijs, \&

E. P. J. van den Heuvel, Cambridge University Press, 1995

Mauche, C.W., ApJ, 464, L87, 1996.

Méndez, M., and M. van der Klis, MNRAS, 318, 938, 2000a.

Méndez, M., M. van der Klis, and E. Ford, ApJ, submitted (astro-ph/0006245) 2000b.

Miller, M. C., ApJ, 531, 458, 2000a.

Miller, M. C., astro-ph/0007287) 2000b.

Miller, M. C., F. K. Lamb, and D. Psaltis, ApJ, 508, 791, 1998.

Patterson, J., ApJS, 45, 517, 1981.

Perez, C. A., A. S. Silbergleit, R. Wagoner, and D. Lehr, ApJ, 476, 589, 1997

Psaltis, D., ApJ, submitted (astro-ph/0010316), 2000.

Psaltis, D., T. Belloni, and M. van der Klis, ApJ, 520, 262, 1999.

Psaltis, D., and C. Norman, ApJ, submitted (astro-ph/0001391), 2000.

Stella, L., M. Vietri, M., and S. Morsink, ApJL, 524, L63, 1999

Strohmayer, T. E., et al., ApJL, 469, L9, 1996.

Strohmayer, T. E., et al., ApJL, 498, L135, 1998.

van der Klis, M., AREA A, in press, 2000.

Wagoner, R.W., Phys. Rep., in press, astro-ph/9805028), 2000.

Zhang, W., et al., ApJ, 500, L171, 1998. 